\title{
Factors Influencing the Sustainability of Neglected Tropical Disease Elimination Programs: A Multi-Case Study of the Kenya National Program for Elimination of Lymphatic Filariasis
}

\author{
Irene Wangeci Thuo, ${ }^{*}$ Khadidiatou Ndiaye, and Sangeeta Mookherji \\ Department of Global Health, Milken School of Public Health, The George Washington University, Washington, District of Columbia
}

\begin{abstract}
Elimination of a disease is the holy grail in global health. The pathology of several neglected tropical diseases (NTDs) such as lymphatic filariasis (LF) makes elimination a reality. However, successful elimination requires that NTD programs be sustainable - the ability to confirm that the disease has been eliminated and the capacity to ensure that it does not return. The WHO's guidelines on NTDs thoroughly detail how to reach elimination. Notwithstanding, comprehensive guidance regarding contextual and programmatic factors that influence sustainability is lacking. Moreover, a comprehensive NTD sustainability framework that includes these factors is nonexistent. This research aimed to develop a framework that identified the critical programmatic and contextual factors influencing sustainability of NTD elimination programs. The methodology included a literature review and a multiple case study. The literature review had two objectives: the first was to identify unique attributes of NTD elimination programs that should be considered when planning for sustainability and the second was to identify an existing sustainability framework that could be adapted to NTD elimination programs. The literature review resulted in a draft sustainability framework that was then tested, using a multicase study methodology, on the Kenya National Program for Elimination of Lymphatic Filariasis. The result is the first comprehensive NTD sustainability framework that identifies key contextual and programmatic factors that influence NTD sustainability. This framework is an invaluable resource for practitioners and policy makers alike as many countries start to eliminate NTDs.
\end{abstract}

\section{INTRODUCTION}

Lymphatic filariasis (LF) is a neglected tropical disease (NTD) caused by the parasites Wuchereria bancrofti, Brugia malayi, or B. timori. Lymphatic filariasis is transmitted by mosquitoes, the same vector as malaria. ${ }^{1,2}$ The disease causes gross swelling (lymphedema) leading to disability. ${ }^{1}$

There are three strategies to prevent, control, and eliminate LF that should be implemented in combination with each other. The first is an annual dose of albendazole and ivermectin with or without diethylcarbamazine. The second is mosquito control measures such as use of bed nets, and the third is surgical intervention for those with late-stage LF complications. ${ }^{3}$ The WHO asserts that with these strategies in place, LF can be eliminated as a public health problem globally by $2020 .^{4}$

Because LF and malaria are both transmitted by the mosquito, ${ }^{1}$ the $\mathrm{WHO}$ advocates that both diseases use an integrated approach to control mosquitos. More specifically, WHO advocates tracking of the impact of distributing insecticide-treated mosquito nets and indoor residual spraying on reducing the transmission of both diseases. ${ }^{2}$

Sustainability research reveals that the approaches recommended by WHO do not address all the necessary steps that NTD programs must take to sustain program activities and outcomes to ensure disease elimination. For example, the guidance could not address the political instability, violent conflict, and large-scale internal displacement in the Democratic Republic of the Congo that led to the complete suspension of NTD efforts. ${ }^{5}$ For purposes of this research, sustainability is defined as a program's ability to continuously and effectively reduce LF prevalence while being responsive and adaptive to changes in the technical guidelines from the

*Address correspondence to Irene Wangeci Thuo, George Washington University, 1515 Jefferson Davis Hwy, Arlington, VA 22202.

E-mail: ithuo@gwu.edu
WHO, programmatic changes within the LF program and the context within which the program is implemented. ${ }^{6}$

An estimated 3.7 million Kenyans are living in areas endemic for LF and, therefore, require regular treatment. ${ }^{7}$ The Kenyan government started the LF treatment in 2002, but individuals have received treatment only six times over a period of 13 years. ${ }^{4}$ Despite these challenges, studies show that the prevalence of LF decreased from $20.9 \%$ in $2002-0.9 \%$ in $2009,{ }^{8}$ making the Kenya LF program a suitable program for this research. Kenya was also chosen as a case study because of existing technical, programmatic, and contextual factors that were identified in the development of the sustainability framework. For example, the National Program for Elimination of Lymphatic Filariasis (NPELF) interventions was impacted by inconsistent treatment because of lack of donor funding over several years (technical factor). The ratification of a new constitution in 2010 (identified in the results as devolution) meant that the NPELF program had to adjust to split health responsibilities between the national and local governments and the introduction of county-led treatment (programmatic). Furthermore, given the longs standing marginalization of several communities in Kenya for which the NPELF operates and the differing cultural practices of those communities, the likelihood of contextual factors being present was high. These realities did not make the research determinative but rather ensured a relevant case study to begin the exploration of these types of factors.

This research, therefore, identified the programmatic and contextual factors that enable or constrain the sustainability of an NTD programs using the LF elimination program as the example. Furthermore, the research explores the practical integration of the malaria and LF programs.

\section{METHODS}

This research was conducted in two steps: a literature review and a multiple case study. The literature review had two objectives: to identify the unique attributes of NTD 
elimination programs that should be considered when planning for sustainability and then to identify an existing sustainability framework that could both be adapted to the NTD space and reflect these unique attributes.

A search was conducted on Scopus, Plos NTD, Google Scholar, MEDLINE, Cochrane, and the WHO Web site using the search terms: Iymphatic filariasis, elephantiasis, elimination, control, program, onchocerciasis, river blindness, trachoma, schistosomiasis, soil-transmitted helminths, intestinal worms, and bilharzia. In 1997, the WHO declared LF as a global target for elimination as a public health problem. Therefore, articles written between 1997 and 2016 were selected. Articles also needed to be published in English and are available in full text. A total of 29 articles were reviewed.

A search was also conducted on Scopus, MEDLINE (Bethesda, MD), and Cochrane using the search terms: neglected tropical diseases, sustainability, financial sustainability, institutionalization, public health programs, and program transition. Two public health experts also recommend existing articles on sustainability frameworks that were not identified in the original two searches. Articles written in English and available in full text were included. A total of 14 articles presenting various sustainability frameworks were reviewed. Eight of these articles were further reviewed to assess the viability of being adopted for NTD programs based on their flexibility to respond to the unique attributes of NTD elimination programs identified in the aforementioned objective. Two of these frameworks were identified as relevant (see Figure 1 for the flow diagram on selection of frameworks). These two frameworks pre-identified the contextual and programmatic factors that could influence NTD sustainability. These factors served as the a priori themes and subthemes used in the multiple case study analysis

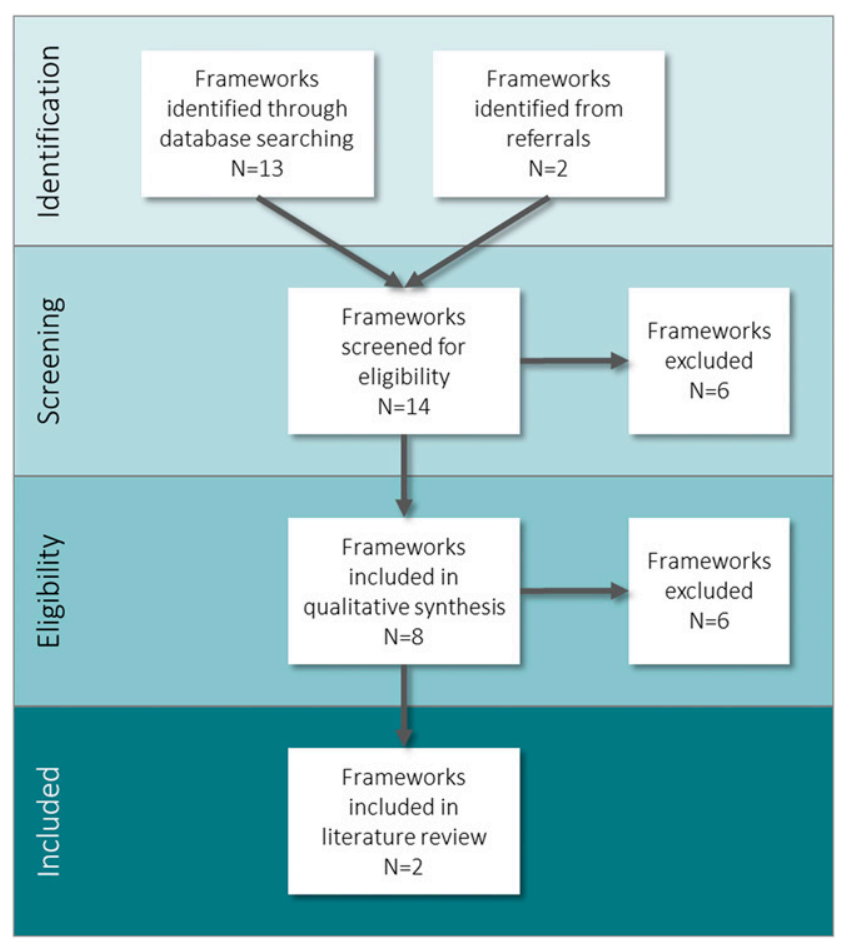

FIGURE 1. Flow diagram for the selection of sustainability frameworks. This figure appears in color at www.ajtmh.org. described in the following paragraphs. The two frameworks were overlaid with the findings from the 29 articles to develop a draft NTD Sustainability Framework shown in Figure 2.

A multiple case study was used to test the draft NTD Sustainability Framework. The Kenya NPELF was identified as a suitable case study having reduced the overall prevalence rate despite having to stop and restart its treatment programs. Three counties, each with an active LF elimination program, were selected.

The framework was tested by triangulating data from 24 semi-structured individual and group interviews that also included participants from the malaria sector. The interview guide in Supplemental Appendix 1 was used for this. Additional data sources included a review of program documents and observation of key meetings. All data, including interview transcripts, program documents, and notes from observations, were typed and entered into NVivo Pro 11, a qualitative data analysis software, for coding and analysis.

Data analysis was conducted by first identifying the a priori and new themes and subthemes within each of the data source. Next, a priori and new themes and subthemes across all three sources were identified. Finally, a priori and new themes and subthemes were compared between the malariaspecific sources and NTD-specific sources.

\section{RESULTS AND DISCUSSION}

The first step of the literature review demonstrated that NTD elimination programs have three phases: start and scale up of program (Phase 1), maintenance of treatment (Phase 2), and scale down and surveillance (Phase 3). Critical to this research was the exploration of not only which programmatic and contextual factors influence sustainability overall but also at what phase of the NTD elimination program are each of them relevant.

The second step of the literature review identified two frameworks from Shigayeva and Coker and Shediac-Rizkallah and Bone $e^{6,9}$ that were found to be relevant and adaptable to NTD elimination programs and identified three categories of factors influencing the sustainability of NTD programs: 1) technical factors, or factors related to the actual intervention; 2) programmatic factors, those related to the implementation of the program; and 3) contextual factors, or factors related to the environment in which the program is being implemented.

By overlaying the findings from both literature reviews, the draft NTD sustainability framework (Figure 2 showed that contextual factors influencing the sustainability of NTD elimination program are 1) political commitment that the program gets, particularly from influential individuals who are termed "champions," 2) reliable funding from diversified sources of funding for the program, including specific contributions from the government, which should increase over time, 3) political stability in the country to provide a safe environment for the program to operate, and 4) environmental improvement in terms of improved water and sanitation facilities.

The framework also showed that programmatic factors influencing the sustainability of NTD elimination programs are 1) leadership capabilities of program's actors to lead, govern, and manage the program; 2) capacity, including, managerial, technical, and financial skills of those working in the program which enable the program to function over time; 3) flexibility/ adaptability of actors to adapt in response to external 


\section{Original Sustainability Model}

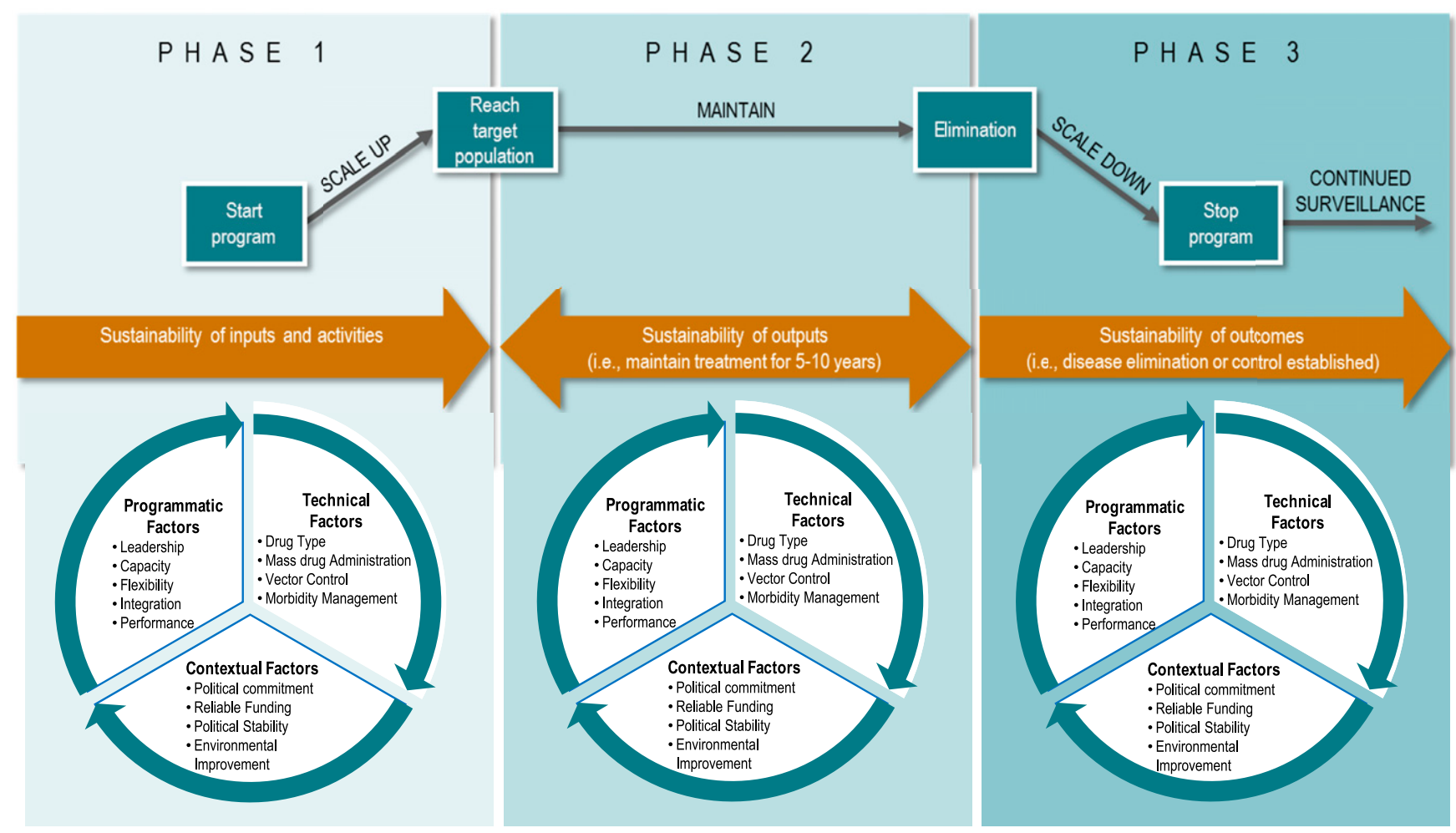

FIGURE 2. Draft neglected tropical disease sustainability framework. This figure appears in color at www.ajtmh.org.

pressures, changes in nature of a disease, or introduction of innovations; and 4) integration of the program with other agencies to support the program. In addition, the relative influence of each factor was dependent on the phase of NTD elimination. The multiple case study showed that contextual factors were more relevant to sustainability than programmatic factors. Among contextual factors, reliable funding and governance systems were most relevant. Five new contextual subthemes emerged: cultural beliefs, education levels, lifestyle, urban/rural settings, and governance. Among programmatic factors, integration of the LF program into the broader health system, and not just into the malaria program, was as a key factor influencing sustainability. A new subtheme within the programmatic factors was the role of the community, particularly the community drug distributers and the beneficiaries of treatment, in sustaining the program. Planning for sustainability is best undertaken in the design phase. Anew NTD sustainability framework was then developed and is shown in Figure 3.

Limitations. A comprehensive sustainability framework for NTDs does not exist, and, therefore, no baseline is available for comparison. However, this was mitigated by adapting a framework from a relevant disease elimination program and including data from countries and programs that have achieved elimination. The research was heavily reliant on individual and group interviews, which presents several limitations, including potential for bias as the interviewees are purposely selected. Specific to group interviews, there was reduced time to have in-depth discussions and limitations in freedom of expression, this latter point was especially relevant as the group interviews in this research were with government employees for whom hierarchy is particularly important. All these limitations are mitigated by the inclusion of interviewees who represented various professional and geographic areas from health and development areas.

\section{CONCLUSION}

Overall, this research's findings support the following key outcomes:

1. Although there remain three categories of factors that influence sustainability, contextual factors are more relevant than programmatic factors.

2. Reliable funding remained a major contextual factor influencing sustainability.

3. Government systems were a new factor identified in this research. It is a factor that has a significant influence on sustainability just after reliable funding. Other new contextual factors included rural-urban environment, education 

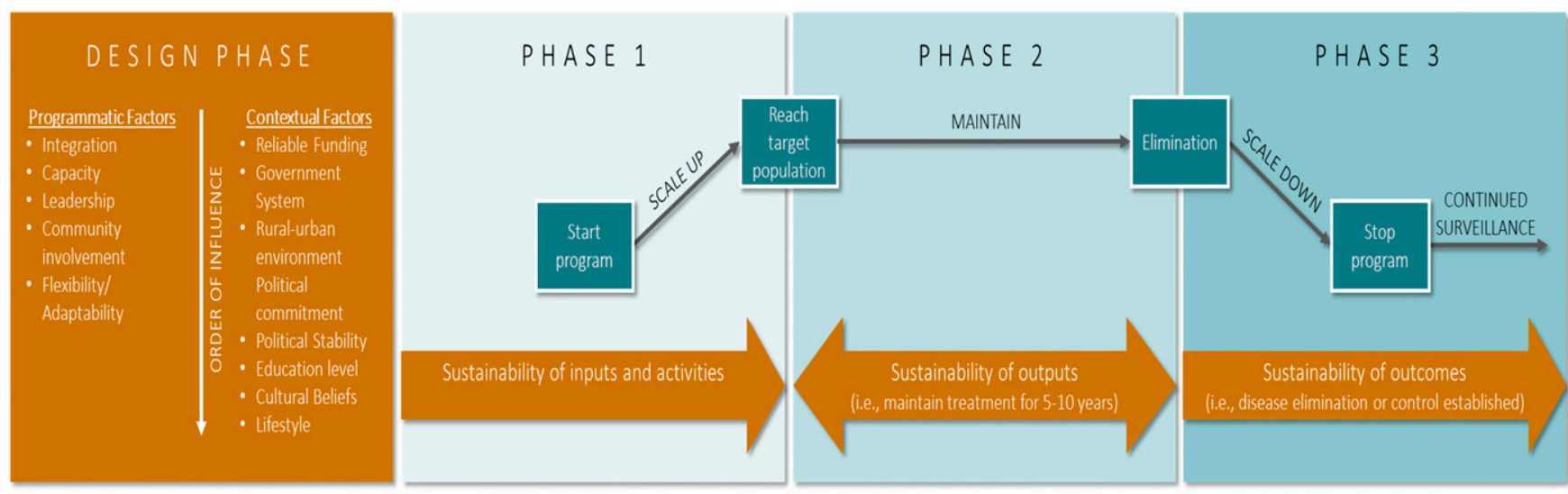

FIGURE 3. Comprehensive sustainability framework for neglected tropical diseases. This figure appears in color at www.ajtmh.org.

level, cultural beliefs, and lifestyle, which had minimal influence on sustainability planning.

4. The contextual factor of performance was not found to be a major factor.

5. Among the programmatic factors, integration of the LF program into existing programs was considered important. This integration, however, did not necessarily extend into the Malaria program.

6 . The role of the community was identified as a new programmatic factor; it was, however, not among the most relevant.

An opportunity for further research exists in analysis of the role of governance systems and community involvement in in sustainability efforts. In addition, further research on the integration of LF programs into existing health systems would be beneficial.

Received April 5, 2019. Accepted for publication November 23, 2019. Published online March 2, 2020.

Note: Supplemental Appendix appears at www.ajtmh.org.

Acknowledgments: This research was made possible by the work of others before me. I am grateful for the path they have created for me. I thank Peter Hotez for the mentorship he has provided throughout my career and in realizing this research. I thank Ketura Brown for being an unwavering source of encouragement throughout this research. I also thank my family and friends who have been a part of this research journey.

Financial support: This study was partially supported by the George Washington University, Milken School of Public Health.

Authors' addresses: Irene Wangeci Thuo, Khadidiatou Ndiaye and Sangeeta Mookherji, Department of Global Health, Milken School of Public Health, The George Washington University, Washington, DC, and Department of Global Health, Washington, DC, E-mails: ithuo@ gwu.edu,kndiaye@gwu.edu and smookherji@gwu.edu.

\section{REFERENCES}

1. World Health Organization, 2013. Sustaining the Drive to Overcome the Global Impact of Neglected Tropical Diseases. Second
WHO Report on Neglected Diseases. Geneva, Switzerland: World Health Organization. Available at: https://books.google. co.za/books?hl=en\&lr=\&id=YrlXDAAAQBAJ\&oi=fnd\&pg=PP1\& $\mathrm{dq}=$ Sustaining+the+drive+to+overcome+the+global+impact+ of+neglected+tropical+disease\&ots=dVDuwGYDjW\&sig= bBybyLO-wM3Oadp8XdBu4frAzbs\&redir_esc $=y \# v=$ onepage\& q=Sustaining\%20the $\% 20$ drive $\% 20$ to $\% 20$ overcome $\% 20$ the $\%$ 20global\%20impact\%20of\%20neglected\%20tropical\%20disease\& $\mathrm{f}=$ false.

2. World Health Organization, 2011. Position statement on integrated vector management to control malaria and lymphatic filariasis. Wkly Epidemiol Rec 86: 121-127.

3. Gyapong JO, Twum-Danso NA, 2006. Editorial: global elimination of lymphatic filariasis: fact or fantasy? Trop Med Int Health 11: 125-128.

4. World Health Organization, 2017. Integrating Neglected Tropical Diseases into Global Health and Development: Fourth WHO Report on Neglected Tropical Diseases. Geneva, Switzerland: World Health Organization. Available at: https://apps.who.int/ iris/bitstream/handle/10665/255011/9789241565448-eng.pdf? sequence $=1$.

5. Okeibunor J, Bump J, Zouré HM, Sékétéli A, Godin C, Amazigo UV, 2012. A model for evaluating the sustainability of communitydirected treatment with ivermectin in the African Program for Onchocerciasis Control. Int J Health Plann Manage 27: 257-217.

6. Shigayeva A, Coker RJ, Scheirer MA, Dearing JW, ShediacRizkallah MC, Bone LR, 2015. Planning for the sustainability of community-based health programs: conceptual frameworks and future directions for research, practice and policy. Health Educ Res 13: 368-385.

7. World Health Organization, 2000. Lymphatic filariasis. Lymphat Filariasis: 2017-2019. Available at: https://books.google.co.za/ books?hl=en\&lr=\&id=OgC4CgAAQBAJ\&oi=fnd\&pg=PA103\& $\mathrm{dq}=$ Lymphatic+Filaraisis\&ots=_szv-mbwLa\&sig=8_SEX5dJ_ kcxNuST67S8iyz4A8s.

8. Njenga SM, Mwandawiro CS, Wamae CN, Mukoko DA, Omar AA, Shimada M, Bockarie MJ, Molyneux DH, 2011. Sustained reduction in prevalence of lymphatic filariasis infection in spite of missed rounds of mass drug administration in an area under mosquito nets for malaria control. Parasit Vectors 4: 90.

9. Shediac-Rizkallah MC, Bone LR, 1998. Planning far the sustainability of community-based health programs: conceptual frameworks and future directions for research, practice and policy. Health Educ Res 13: 87-108. 